\title{
On the Oscillatory Behaviour of a Second Order Nonlinear Differential Equation $\left(^{*}\right)\left(^{* *}\right)$.
}

\author{
G. J. Butler (Edmonton, Canada)
}

Summary. - The equation $y^{\prime \prime}(t)+p(t) f(y(t))=0$ is considered, in which $f(y)$ has the same sign as $y$, is monotone increasing, and is "strongly nonlinear 》, and $p(t)$ is locally integrable such that $\lim _{T \rightarrow \infty} \int_{i}^{T} p(s)$ ds exists. No sign condition is assumed, either on $p$, or its integral. Under conditions which guarantee that, in a certain sense, $p$ is not too negative, an integrability condition is obtained which is necessary and sufficient for all extendable solutions to oscillate. In the second half of the paper, the monotonicity and strong nonlinearity assumptions on $f$ are shown to be essential ingredients for the existence of such osillatory eriteria. Some exam. ples illustrating the theorems are given.

\section{1. - Introduction.}

The question of oscillation of the solutions of

$$
y^{\prime \prime}(t)+F(t, y(t))=0
$$

has received the attention of a number of authors since the original result of ATKINson [1] who, considering the special case

$$
y^{n}(t)+p(t) y^{2 n-1}=0
$$

where $p(t)>0$ and continuous and $n \geqslant 2$, obtained the divergence of $\int_{i}^{\infty} s p(s) d s$ as a necessary and sufficient condition for the oscillation of all solutions. (1.2) was also considered by Moone and NeHARr [9] and UTz [11, 12], and the more general

$$
y^{\prime \prime}(t)+p(t) f(y(t))=0
$$

has received the attention of (amongst others) WALTMaN [13], NEHARr [10] and Wong [17]. Mack and WoNG [8] obtained a result which extended that of Atrursson to (1.3) when $f(y)$ is "strongly nonlinear ".

(*) Entrata in Redazione l'8 agosto 1973.

(**) Research supported in part by NRC grant no. A-8130. 
In [13], WALTMAN, and later ERBE in [4] related the oscillatory character of (1.3) to that of the linear equation

$$
y^{\prime \prime}(t)+p(t) y(t)=0 .
$$

The first oscillation result for $(1.2)$ in which $p(t)$ is no longer assumed to be nonnegative seems to have been that of WALTMan [14] who showed that $\int_{t}^{\infty} p(s) d s=+\infty$ implies that all solutions of (1.3) oscillate.

In [3], CoLes obtained a necessary condition and a (different) suffieient condition for all solutions of (1.1) to oseillate which applied to (1.3) without the assumption of non-negativity on $p(t)$. We state here his results as they apply to (1.3).

Theorem 1.1. - Let $f(y)$ and $p(t)$ satisfy the following conditions:

$\mathscr{F}_{1}: f(y)$ is absolutely continuous with $y f(y)>0$ for $y \neq 0$ and $f^{\prime}(y) \geqslant 0$ for almost all $y$.

$$
\mathscr{F}_{2}: \int_{ \pm 1}^{+\infty} \frac{d y}{f(y)}<\infty
$$

$\mathfrak{J}_{1}: \quad p(t)$ is locally integrable with $0 \leqslant \int_{t}^{\infty} p(s) d s=P(t)$ for all sufficiently large $t$

Then $\int_{i}^{\infty} P(s) d s=\infty$ implies that all extendable solutions of (1.3) oseillate.

[Here, and in the sequel, "extendable means extendable to a right half-line].

THEOREM 1.2. - Let $p(t)$ satisfy $\mathfrak{T}_{1}$ and let $f(y)$ satisfy:

$\mathscr{F}_{3}$ : there exists a non-trivial compact interval $I$ in which $y f(y)>0$ for $y \neq 0$ and $f^{\prime}(y)$ exists at each point, is non-negative and bounded.

Then $\int_{i}^{\infty} \int_{s}^{\infty} P(\sigma) d \sigma d s<\infty$ implies that there is a non-trivial non-oseillatory solution of (1.3).

The main object of this paper is to obtain a necessary and sufficient condition for the oscillation of all solutions of (1.3) under essentially the same conditions as those imposed by COLEs.

As corollaries, we shall obtain a characterization of those coefficients $p(t)$ for which the equation (1.4) has a bounded oscillatory solution, and extend a result of ERBE [4] relating oscillation coefficients for (1.4) to those for (1.3).

We shall then investigate the extent to which the conditions imposed on $f$ are needed; in particular we are able to answer a question raised by WoNG in [16] and partially solved by MAcKr [7].

In a future paper, we hope to extend our results to the equation

$$
y^{\prime \prime}(t)+q(t) g\left(y^{\prime}(t)\right)+p(t) f(y(t))=0 .
$$


Finally, we refer the reader to the survey article [17] and the extensive bibliography therein.

We should also add that by a solution of (1.3) ete. we shall in general mean a solution in the Carathéodory sense, since $p(t)$ is not assumed to be continuous.

\section{2. - Oscillation theorems for $(1.3)$.}

Before stating the results of this section, we introduce the notation $g_{+}, g_{-}$for any real-valued function $g(t)$

$$
g_{+}(t)=\max (g(t), 0), \quad g_{-}(t)=g_{+}(t)-g(t) .
$$

Our main result will be the following:

Theorem 2.3. - Let $f(y)$ and $p(t)$ satisfy the hypotheses

$\Phi_{1}$ : $f(y)$ is absolutely continuous on any bounded set with $y f(y)>0$, for $y \neq 0$, and the essential infimum of $f^{\prime}(y)$ over any closed set that excludes zero is positive.

$\Phi_{2}: \int_{ \pm 1}^{ \pm \infty} \frac{d y}{f(y)}<\infty$.

$\Phi_{3}$ : there is a non-trivial compact interval $I$ in which $y f(y)>0$ for $y \neq 0$ and $f^{\prime}(y)$ exists at each point, is non-negative and bounded.

$\pi_{1}: \quad p(t)$ is locally integrable and $\int_{i}^{\infty} p(s) d s=P(t)$ exists (possibly infinite) with $\varliminf_{T \rightarrow \infty} \int_{i}^{T} P(s) d s>-\infty$ for all $t$.

$\pi_{2}: \quad \int_{i}^{\infty}\left(\int_{s}^{\infty} P_{-}^{2}(\sigma) d \sigma\right) d s<\infty$

Then all extendable solutions of (1.3) oseillate if and only if

$$
\int_{i}^{\infty}\left[P(s)+\int_{s}^{\infty} P^{2}(\sigma) d \sigma\right] d s=\infty .
$$

Theorem 2.3 will be a consequence of the following two results:

Theorem 2.2. - Let $f(y)$ and $p(t)$ satisfy $\Phi_{1}, \Phi_{2}$ and $\pi_{1}$. Then

$$
\int_{i}^{\infty}\left[P(s)+\int_{s}^{\infty} P_{+}^{2}(\sigma) d \sigma\right] d s=\infty
$$

implies all extendable solutions of (1.3) oscillate. 
THEonem 2.2. - Let $f(y)$ and $p(t)$ satisfy $\Phi_{3}, \pi_{1}$ and $\pi_{2}$. Then

$$
\int_{i}^{\infty}\left[P(s)+\int_{s}^{\infty} P^{2}(\sigma) d \sigma\right] d s<\infty
$$

implies that there is a non-trivial, non-oscillatory solution of (1.3). We remark that $\left(\pi_{1}\right)$ and $\left(\pi_{2}\right)$ are, in particular, satisfied if $P(t) \geqslant 0$ for sufficiently large $t$ (condition $\left(T_{1}\right)$ ). The additional condition in $\Phi_{1}$ on the behaviour of $f^{\prime}$ cannot be completely omitted as we shall indicate in section 3 .

Theorems 2.1 and 2.2 will be proved under the stronger assumption that $f$ is $C^{1}$ rather than absolutely continuous, with the ensuing consequences for $\left(\Phi_{1}\right)$ and $\left(\Phi_{3}\right)$. This is to avoid unnecessary technical complications that arise in the proof if $f^{\prime}$ is not defined everywhere. An examination of the arguments used will show that the only places in which absolute continuity of $f$, rather than $C^{1}$ behaviour, requires a non-routine modification of the proof are in the differentiation of $f(y(t))$. If $f$ is absolutely continuous and $y(t)$ is $C^{1}$, then $f(y(t))$ will be absolutely continuous, and so differentiable almost everywhere, and the statement

$$
y^{\prime}(t) \frac{d}{d t} f(y(t))=f^{\prime}\left((y(t)) y^{\prime 2}(t), \quad \text { for all } t\right.
$$

can be replaced by

$$
y^{\prime}(t) \frac{d}{d t}\left(f(y(t))=\varphi(y(t)) y^{\prime 2}(t), \quad \text { for almost all } t\right.
$$

where

$$
\varphi(u)= \begin{cases}f^{\prime}(u), & \text { if this exists } \\ 1, & \text { otherwise }\end{cases}
$$

and the proofs may be carried out with no other essential changes.

Proof of THEOREM 2.1. - Let the conditions of the theorem be satisfied and suppose that $y(t), t \geqslant t_{0}$, is a non-oseillatory solution which, without loss of generality, we shall assume to be positive. Define $z(t)$ to be $y^{\prime}(t) / f(y(t))$ to obtain the Riccati equation

$$
z^{\prime}(t)=-p(t)-f^{\prime}(y(t)) z^{2}(t)
$$

Then, for $t_{0} \leqslant t \leqslant T$,

$$
z(t)=z(T)+\int_{i}^{T} p(s) d s+\int_{i}^{T} f^{\prime}(y(s)) z^{2}(s) d z
$$


We consider two cases:

(i) $\varlimsup_{T \rightarrow \infty} \int_{i}^{T} P(s) d s=+\infty \quad$ for some $t \geqslant t_{0}$;

(ii) $\varlimsup_{T \rightarrow \infty} \int_{i}^{T} P(s) d s<\infty \quad$ for all $t \geqslant t_{0}$.

Case (i). We note that $\varlimsup_{T \rightarrow \infty} \int_{t}^{T} P(s) d s=+\infty \quad$ for all $t \geqslant t_{\lrcorner}$.

If there is a sequence $T_{n} \uparrow \infty$ such that $z\left(T_{n}\right) \geqslant 0$, we have from (2.2)

$$
z(t) \geq P(t)+\int_{i}^{\infty} f^{\prime}(y(s)) z^{2}(s) d s
$$

so that $z(t) \geqslant P(t)$. Integrating from $t_{0}$ to $T$, we obtain

$$
\int_{y\left(t_{0}\right)}^{y(T)} \frac{d u}{f(u)} \geqslant \int_{t_{0}}^{T} P(s) d s
$$

and taking the $\lim _{T \rightarrow \infty}$ sup on both sides, we obtain a contradiction on account of $\left(\Phi_{2}\right)$.

If there is no such sequence $T_{n}$, then

$$
z(t)<0 \quad \text { for all } t \geqslant T_{0},
$$

say. Thus

$$
y^{\prime}(t)<0 \quad \text { for all } t \geqslant T_{0} .
$$

Following Coles, we define

$$
t_{i}=\inf \left\{t \geqslant T_{0}: y^{\prime}(t)=-\frac{1}{i}\right\}, \quad i=1,2, \ldots
$$

Then for $i$ sufficiently large, $t_{i}$ is well-defined (otherwise $y^{\prime}(t)$ would be bounded above by some negative number and $y(t)$ could not remain positive) and $t_{i} \uparrow \infty$. Defining $F(t)=f(y(t)) \int_{t}^{t_{t}} p(s) d s$, we obtain (see [3])

$$
F(t)<0 \quad \text { on } \quad\left[t_{i-1}, t_{i}\right) .
$$

6 - Annali di Matematica 
So we have $\int_{i}^{t} p(s) d s<0$ for $t \in\left[t_{i-1}, t_{i}\right)$. Thus

$$
\begin{aligned}
P(t) & =\lim _{k \rightarrow \infty} \int_{i}^{t_{k}} p(s) d s \\
& =\lim _{k \rightarrow \infty}\left[\int_{t}^{t_{i}} p(s) d s+\sum_{j=1}^{k-1} \int_{t_{i}}^{t_{j+1}} p(s) d s\right]<0
\end{aligned}
$$

and so $P(t)<0$ for sufficiently large $t$, clearly a contradiction.

Case (ii). Note that in this case, we shall have for each $t \geqslant t_{0}$,

$$
\left|\int_{i}^{T} P(s) d s\right| \quad \text { uniformly bounded for } T \geqslant t
$$

(the bound will depend on $t$ ) and

$$
\int_{i}^{\infty} \int_{s}^{\infty} P_{+}^{2}(\sigma) d \sigma d s=+\infty
$$

If there is a sequence $T_{n} \uparrow \infty$ such that $z\left(T_{n}\right) \geqslant 0$, then (2.2) yields, as before, $z(t) \geqslant$ $\geqslant P(t)+\int_{i}^{\infty} f^{\prime}(y(s)) z^{2}(s) d s$, so that $z(t) \geqslant P(t)$ and therefore $z^{2}(t) \geqslant P_{+}^{2}(t)$, giving

$$
z(t) \geqslant P(t)+\int_{i}^{\infty} f^{\prime}(y(s)) P_{+}^{2}(s) d s
$$

If $y(t) \geqslant \delta>0$ for all large $t$, then let $\varepsilon=\inf _{\delta \leqslant t<\infty} f^{\prime}(u)$, which is positive by $\left(\Phi_{1}\right)$. Then

$$
z(t) \geqslant P(t)+\varepsilon \int_{t}^{\infty} P_{+}^{2}(s) d s, \quad \text { for large } t
$$

Integrating (2.4) we obtain

$$
\int_{\nu(\delta)}^{y(T)} \frac{d u}{f(u)} \geqslant \int_{i}^{T} P(s) d s+\varepsilon \int_{i}^{T} \int_{s}^{\infty} P_{+}^{2}(\sigma) d \sigma d s,
$$

and letting $T \rightarrow \infty$ we obtain a contradiction. If $y(t)$ is not bounded below, let 
$T_{n}^{\prime} \downarrow \infty$ with $y\left(T_{n}^{\prime}\right) \downarrow 0$. If $n$ is sufficiently large, we have

$$
0 \geqslant \int_{y\left(t_{0}\right)}^{y\left(T_{n}^{\prime}\right)} \frac{d u}{f(u)}=\int_{t_{0}}^{T_{n}^{\prime}} z(s) d s \geqslant \int_{t_{0}}^{T_{\sharp}^{\prime}} P(s) d s+\int_{t_{0}}^{T_{0}^{\prime}} \int_{s}^{\infty} f^{\prime}(y(\sigma)) P_{+}^{2}(\sigma) d \sigma d s,
$$

by (2.3).

If $f^{\prime}(0)>0,\left(\Phi_{1}\right)$ implies that $\inf _{u<0} f^{\prime}(u)>0$ and letting $n \rightarrow \infty$ we obtain a contradiction, since the right hand side of $(2.5)$ tends to $+\infty$.

Hence we may suppose that $f^{\prime}(0)=0$ and so we have

$$
0 \leqslant \int_{0}^{y\left(t_{0}\right)} \frac{d u}{f(u)} \leqslant-\lim _{T \rightarrow \infty} \int_{t_{a}}^{T} P(s) d s<+\infty,
$$

by $\left(\pi_{1}\right)$, which will be a contradiction of $f(0)=f^{\prime}(0)=0$. Finally, if there is no such sequence $T_{n}$, we may argue as in case (i) for this situation, and the proof of the theorem is concluded.

Proof of Theorem 2,2. - Let the hypotheses of the theorem hold, so that $\int_{i}^{\infty}\left[[P(s)]+\int_{s}^{\infty} P^{2}(\sigma) d \sigma\right] d s<\infty\left(P\right.$ is absolutely integrable on account of $\left.\left(\pi_{1}\right)\right)$.

We shall make use of the Schauder fixed point theorem to demonstrate the existence of a (non-trivial) non-oseillatory solution to (1.3).

Without loss of generality, let $I=[\alpha, \beta]$ with $0<\alpha<\beta$, and choose positive constants $e, \varepsilon$ with $e>\varepsilon$ such that $[c-\varepsilon, e+\varepsilon] c[\alpha, \beta]$.

We define the constants $A, A_{1}, B$ and the function $Q(t)$ by

$$
\begin{aligned}
& A=\sup _{\alpha \leqslant u \leqslant \beta} f(u), \\
& B=2 A A_{1},
\end{aligned}
$$

$$
\begin{aligned}
& A_{1}=\sup _{\alpha \leqslant u \leqslant \beta} f^{\prime}(u), \\
& Q(t)=A|P(t)|+B \int_{t}^{\infty} P^{2}(s) d s .
\end{aligned}
$$

Now we choose $T>0$ so large that

$$
A_{1} \int_{T}^{\infty}|P(s)| d s \leqslant \frac{1}{2}
$$

$$
A_{1} Q(s)\left|\int_{s}^{t} p(\sigma) d \sigma\right| \leqslant 1
$$

for all $s, t>T$, and

$$
\int_{T}^{\infty} Q(s) d s \leqslant \varepsilon
$$


Define $\delta$ to be the space of bounded, absolutely continuous functions on $[T, \infty)$ whose derivatives are almost everywhere bounded in absolute value by some multiple of $Q(t)$.

With \|\|$_{\infty}$ denoting the uniform norm, we make $S$ into a Banach space by introducing the norm

$$
\|y\|=\|y\|_{\infty}+\left\|Q^{-1} y^{\prime}\right\|_{\infty}
$$

For $n=1,2, \ldots$, define $\mathfrak{B}_{n}$ to be the subset of functions $y$ in $\delta$ such that

$$
\begin{gathered}
\|y-c\|_{\infty} \leqslant \varepsilon, \quad\left\|Q^{-1} y^{\prime}\right\|_{\infty} \leqslant 1 . \\
\left|y^{\prime}\left(t_{2}\right)-y^{\prime}\left(t_{1}\right)\right| \leqslant A\left|\int_{t_{2}}^{t_{3}} p(s) d s\right|+\left|t_{2}-t_{1}\right|,
\end{gathered}
$$

for all $t_{1}, t_{2} \in[T, T+n)$, and

$$
y \quad \text { is constant for } t \geqslant T+n \text {. }
$$

Then $\mathscr{B}_{n}$ is a convex subset of $\mathcal{S}$, and by the Ascoli theorem, $\mathscr{B}_{n}$ is compact.

Fof $y \in \mathfrak{B}_{n}$, define the map $T_{n}$ by

$$
\left(T_{n} y\right)(t)= \begin{cases}c-\int_{i}^{\infty} \int_{s}^{\infty} p(\sigma) f(y(\sigma)) d \sigma d s, & T \leqslant t<T+n \\ c-\int_{T+n}^{\infty} \int_{s}^{\infty} p(\sigma) f(y(\sigma)) d \sigma d s, & T+n \leqslant t .\end{cases}
$$

We note that since $y(t) \in \mathfrak{B}_{n}$ is constant on $[T+n, \infty)$, the integrability of $p$ and $|P|$ imply that $T_{n}$ is a well-defined map of $\Re_{n}$ at least into the space of absolutely continuous functions with a.e. bounded derivatives.

We shall in fact verify that $T_{n}$ is a continuous map of $\mathfrak{B}_{n}$ into itself.

Let $y \in \mathscr{B}_{n}$. Then for $T \leqslant t<T+n$,

$$
\left(T_{n} y\right)^{\prime}(t)=\int_{t}^{\infty} p(s) f(y(s)) d s=f(y(t)) P(t)+\int_{i}^{\infty} f^{\prime}(y(s)) P(s) y^{\prime}(s) d s
$$

Thus

$$
\left|\left(T_{n} y\right)^{\prime}(t)\right| \leqslant A|P(t)|+\int_{i}^{\infty} A_{1}|P(s)|\left\{A|P(s)|+B \int_{s}^{\infty} P^{2}(\sigma) d \sigma d s,\right.
$$


using (2.6) and (2.7)

$$
\begin{aligned}
& \leqslant A|P(t)|+\frac{1}{2} B \int_{i}^{\infty} P^{2}(s) d s+A_{1} B\left(\int_{i}^{\infty} P^{2}(s) d s\right)\left(\int_{i}^{\infty}|P(s)| d s\right) \\
& \leqslant Q(t), \quad \text { by }(2.10)
\end{aligned}
$$

Therefore $T_{n}\left(\mathscr{B}_{n}\right) \subset \mathcal{S}$. Furthermore, if $T \leqslant t_{1}<t_{2}<T+n$, we have

$$
\begin{aligned}
\left|\left(T_{n} y\right)^{\prime}\left(t_{2}\right)-\left(T_{n} y\right)^{\prime}\left(t_{1}\right)\right| & =\left|\int_{t_{1}}^{t_{3}} p(s) f(y(s)) d s\right| \\
& =\left|f\left(y\left(t_{1}\right)\right) \int_{i_{1}}^{t_{2}} p(s) d s+\int_{i_{1}}^{t_{3}} f^{\prime}(y(s)) y^{\prime}(s)\left(\int_{s}^{t_{1}} p(\sigma) d \sigma\right) d s\right| \\
& \leqslant A\left|\int_{t_{1}}^{t_{2}} p(s) d s\right|+\int_{t_{1}}^{t_{2}} A_{1} Q(s)\left|\int_{s}^{t_{2}} p(\sigma) d \sigma\right| d s \\
& \leqslant A\left|\int_{t_{1}}^{t_{3}} p(s) d s\right|+\left|t_{2}-t_{1}\right|, \quad \text { by (2.11). }
\end{aligned}
$$

For $T \leqslant t \leqslant T+n$, we have

$$
\begin{aligned}
\left|T_{n} y-e\right|(t) & =\left|\int_{t}^{\infty} \int_{s}^{\infty} p(\sigma) f(y(\sigma)) d \sigma d s\right| \\
& \leqslant \int_{i}^{T+n}\left(A|P(s)|+B \int_{s}^{\infty} P^{2}(\sigma) d \sigma\right) d s+\int_{T+n}^{\infty} f(y(T+n))\left|\int_{s}^{\infty} p(\sigma) d \sigma\right| d s \\
& \leqslant \int_{t}^{\infty} Q(s) d s \leqslant \varepsilon, \quad \text { by (2.12) }
\end{aligned}
$$

Since $T_{n} y(t)$ is constant for $t \geqslant T+n$, we have now shown that $T_{n}\left(\mathscr{B}_{n}\right) \subset \mathfrak{B}_{n}$. Now let $y, z \in \mathfrak{B}_{n}$ with $\|y-z\|_{\leqslant} \leqslant \delta(\delta>0)$.

Then, denoting

$$
\sup _{\substack{\alpha \leq u, z \leq c+e \\|u-v| \leqslant \delta}}|f(u)-f(v)| \quad \text { by } a_{0}(\delta)
$$

and

$$
\sup _{\substack{\alpha \leqslant u, v \leqslant c+s \\|u-v| \leqslant \delta}}\left|f^{\prime}(u)-f^{\prime}(v)\right| \quad \text { by } a_{1}(\delta)
$$


we have

$$
\begin{aligned}
& \left|\left(T_{n} y\right)^{\prime}-\left(T_{n} z\right)^{\prime}\right|(t)=\left|\int_{i}^{\infty}(f(y(s))-f(z(s))) p(s) d s\right| \\
& \leqslant|f(y(t))-f(z(t))||P(t)|+\left|\int_{i}^{\infty}\left(f^{\prime}(y(s)) y^{\prime}(s)-f^{\prime}(z(s)) z^{\prime}(s)\right) P(s) d s\right| \\
& \quad \leqslant|f(y(t))-f(z(t))||P(t)|+\int_{i}^{\infty}\left|f^{\prime}(y(s))-f^{\prime}(z(s))\right|\left|y^{\prime}(s)\right||P(s)| d s+ \\
& \quad+\int_{i}^{\infty}\left|f^{\prime}(z(s))\right|\left|y^{\prime}(s)-z^{\prime}(s)\right||P(s)| d s \\
& \leqslant a_{0}(\delta)|P(t)|+\int_{i}^{\infty}\left\{a_{1}(\delta)\left(A|P(s)|+B \int_{s}^{\infty} P^{2}(\sigma) d \sigma\right)+A_{1} \delta\left(A|P(s)|+B \int_{s}^{\infty} P^{2}(\sigma) d \sigma\right)\right\} \\
& \quad \leqslant a_{0}(\delta)|P(t)|+\left(a_{1}(\delta)+\delta A_{1}\right)\left[A \int_{i}^{\infty} P^{2}(s) d s+\left(B \int_{i}^{\infty} P^{2}(s) d s\right)\left(\int_{i}^{\infty}|P(s)| d s\right)\right] \\
& \leqslant a_{0}(\delta)|P(t)|+\left(a_{1}(\delta)+\delta A_{1}\right)(A+\varepsilon B) \int_{i}^{\infty} P^{2}(s) d s \\
& \leqslant b(\delta) Q(t)
\end{aligned}
$$

say, where $b(\delta) \rightarrow 0$ as $\delta \rightarrow 0$. Integrating this last inequality gives

$$
\begin{aligned}
\left|T_{n} y-T_{n} z\right|(t) & \leqslant \int_{i}^{\infty}\left|\left(T_{n} y\right)^{\prime}-\left(T_{n} z\right)^{\prime}\right|(s) d s+\left|T_{n} y-T_{n} z\right|(T+n) \\
& \leqslant b(\delta) \int_{i}^{\infty} Q(s) d s+b_{1}(\delta) \int_{i}^{\infty} Q(s) d s
\end{aligned}
$$

say

$$
\leqslant \varepsilon\left(b(\delta)+b_{1}(\delta)\right)
$$

and so

$$
\left\|T_{n} y-T_{n} z\right\| \leqslant\left(b(\delta)+b_{1}(\delta)((1+\varepsilon)\right.
$$

and it follows that $T_{n}$ is continuous. Now we apply the Schauder fixed point theorem to obtain a function $y_{n} \in \mathfrak{B}_{n}$ such that

$$
T_{n} y_{n}=y_{n}
$$


Thus for $T \leqslant t<T+n$,

$$
y_{n}^{\prime \prime}(t)+p(t) f\left(y_{n}(t)\right)=0
$$

The sequence $\left\{y_{n}\right\}$ is equicontinuous on $[T, \infty)$. It follows by a standard diagonal argument that there is a subsequence $\left\{y_{n_{k}}\right\}$ converging uniformly on compact intervals to a function $y$ which is a non-trivial, bounded non-oscillatory solution on $[T, \infty)$ of $(1.3)$.

REMARK. - For the linear equation (1.4) under the hypothesis $\left(\pi_{1}\right)$ and $\left(\pi_{2}\right)$, the existence of a solution (and hence all solutions) which is non-oseillatory follows very easily from a result of Willett (Theorem 1.1 of [15]); however the boundedness of such a solution (given by Theorem 2.2 above) does not seem to be so evident.

Noting that condition $\left(\Phi_{2}\right)$ is used in the proof of Theorem 2.1 only to deal with unbounded solutions, we have the following

Corollary 2.1. - Let $P(t) \geqslant 0$ and let $f(y)$ satisfy $\left(\Phi_{1}\right)$ and $\left(\Phi_{3}\right)$. Then a necessary and sufficient condition that (1.3) admits a bounded, non-oscillatory solution is that

$$
\int_{i}^{\infty}\left[P(s)+\int_{s}^{\infty} P^{2}(\sigma) d \sigma\right] d s<\infty
$$

Next we give a sufficient condition for oseillation that relaxes the requirement that $\lim _{T \rightarrow \infty} \int_{i}^{T} p(s) d s$ should exist.

THEOREM 2.4. - Let $f(y)$ satisfy $\Phi_{1}$ and $\Phi_{2}$, and let $p(t)$ be locally integrable such that

$\pi_{3}:$ for almost all $t,\left\{T: \int_{i}^{T} p(s) d s>0\right\}$ has infinite measure.

$\pi_{4}$ : there is a sequence $T_{n} \uparrow \infty$ with $\frac{\lim _{T \rightarrow \infty}}{T_{T_{n}}} \int_{0}^{T} p(s) d s \geqslant 0$.

$\pi_{5}:$ for any $\varepsilon>0,\left\{t: \lim _{T \rightarrow \infty} \int_{i}^{T} p(s) d s<-\varepsilon\right\}$ has finite measure.

Let $\hat{P}(t)=\varlimsup_{T \rightarrow \infty} \int_{i}^{T} p(s) d s$. Then a sufficient condition for all solutions of (1.3) to oscillate is that

$$
\int_{i}^{\infty}\left[\hat{P}(s)+\int_{s}^{\infty} \hat{P}^{2}(\sigma) d \sigma\right] d s=\infty
$$


Proof. - The argument follows that of Theorem 2.1. As before, we obtain the Riccati equation (2.2). If $z(t)$ were negative for sufficiently large $t$, we would obtain, as in the proof of Theorem 2.1, a sequence $t_{i} \uparrow \infty$ with $\int_{i}^{t_{i}} p(s) d s<0$ for $t \in\left[t_{i-1}, t_{i}\right)$. This clearly contradicts $\left(\pi_{4}\right)$. Hence there is a sequence $\tau_{n} \uparrow \infty$ with $z\left(\tau_{n}\right) \geqslant 0$. Equation (2.2) then gives

$$
z(t) \geqslant \int_{i}^{\tau_{n}} p(s) d s, \quad \text { for all } t
$$

Using $\left(\pi_{5}\right)$ we have, given $\varepsilon>0$, that $z(t) \geqslant-\varepsilon$ except for a set of finite measure. By $\left(\pi_{3}\right)$, therefore, we may choose for any $t, T>t$ such that

$$
z(T) \geqslant-\varepsilon \quad \text { and } \quad \int_{i}^{T} p(s) d s>0 .
$$

If now follows that $z(t) \geqslant-\varepsilon$ for all $t$. Since $\varepsilon>0$ is arbitrary, we deduce that $z(t) \geqslant 0$ for all $t$ and so, by (2.2),

$$
z(t) \geqslant \hat{P}(t) \quad \text { for all } t
$$

The remainder of the proof now follows directly along the lines of that of Theorem 2.1.

REMARK. - We observe that in showing that $z(t) \geqslant 0$ in the above proof, only the condition $\left(\Phi_{1}\right)$ on $f$ was used.

We may now obtain the following:

COROLLARY 2.2. - Let $p(t)$ satisfy $\left(\pi_{3}\right),\left(\pi_{4}\right)$ and $\left(\pi_{5}\right)$ and let $f(y)$ satisfy $\left(\Phi_{1}\right)$ and e.ther $\left(\Phi_{2}\right)$ or $\left(\Phi_{4}\right): f^{\prime}(x) \geqslant f(x) / x>0$ for $x \neq 0$.

Then if (1.4) is oscillatory, all solutions of (1.3) will oscillate.

Proof. - Assume that (1.4) is oscillatory. Let $\hat{P}(t)$ be $\varlimsup_{T \rightarrow \infty} \int_{i}^{T} P(s) d s$. By $\left(\pi_{s}\right)$ $\hat{P}(t) \geqslant 0$ for almost all $t$. If $\hat{P}(t)$ is finite, we note that

$$
\frac{d}{d t} \hat{P}(t)=-p(t), \quad \text { for almost all } t
$$

Next we define $\bar{P}(t)$ to be

Suppose that

$$
\int_{i}^{\infty} \hat{P}^{2}(s) \exp \left(2 \int_{i}^{s} \hat{P}(\sigma) d \sigma\right) d s .
$$

$$
\int_{i}^{\infty}\left[\hat{P}(s)+\int_{s}^{\infty} \hat{P}^{2}(\sigma) d \sigma\right] d s<\infty
$$


Then

$$
\int_{i}^{\infty} \hat{P}^{2}(s) \exp \left(2 \int_{i}^{s} \hat{P}(\sigma) d \sigma\right) d s \leqslant\left(\int_{i}^{\infty} \hat{P}^{2}(s) d s\right) \exp \left(2 \int_{i}^{\infty} \hat{P}(\sigma) d \sigma\right)
$$

so that

$$
\hat{\boldsymbol{P}}(t)<\infty \text {. }
$$

Furthermore, we see that the non-negativity of $\hat{P}(t)$ ensures that $\bar{P}(t)$ is a non-increasing, non-negative, integrable function of $t$, so that

$$
\begin{aligned}
\int_{t}^{\infty} \bar{P}^{2}(s) \exp 2 & \left(\int_{t}^{s} \hat{P}(\sigma) d \sigma\right) d s \\
& \leqslant \exp \left(2 \int_{i}^{\infty} \hat{P}(\sigma) d \sigma\right) \cdot\left(\int_{i}^{\infty} \bar{P}(s) d s \bar{P}(t)\right) \\
& \leqslant \frac{1}{4} \bar{P}(t), \quad \text { if } t \text { is sufficiently large. }
\end{aligned}
$$

Equations (2.18), (2.20) and (2.21) enable us to appeal to a result of Willett (Theorem 1.1 of [15]) to deduce that (1.4) is disconjugate on the right half line, which is a contradiction. We conclude that $(2.19)$ is false and if $\left(\Phi_{2}\right)$ holds, the oscillation of all solutions of (1.3) follows from Theorem 2.4. If, instead, $\left(\Phi_{4}\right)$ holds, the Remark following Theorem 2.4 indicates that all non-oscillatory solutions $y$ of (1.3) satisfy

$$
y y^{\prime}>0 \quad \text { for sufficiently large } t \text {. }
$$

Applying a result of ERBE [4] we deduce that all non-oscillatory solutions of (1.3) are bounded. Now we may use (2.17) and the non-integrability of $\hat{P}(t)+\int_{t}^{\infty} \hat{P}^{2}(s) d s$ to obtain the required result, noting only that (2.22) implies that $f^{\prime}(y(t))$ is bounded away from zero. This completes the proof of the Corollary.

REMARK. - It is readily seen from the above proof that the condition that (1.4) should be oscillatory may be replaced by the weaker condition that $y^{\prime \prime}+c p(t) y=0$ be oscillatory, for some positive $c$.

\section{3. - The nature of the conditions $\left(\Phi_{1}\right)$ and $\left(\Phi_{2}\right)$.}

We now propose to examine the degree to which the conditions placed on $f$ in the previous section are crucial for oscillation theorems of the type that we have obtained. 
Our first result shows that both the monotonicity condition and the integral condition are pertinent. Specifically, we shall prove

THEOREM 3.1. - Let $f$ be continuously differentiable on $(-\infty, \infty)$ with $y f(y)>0$ for $y \neq 0$. Let $\mathfrak{b}$ be the class of non-negative, locally integrable functions $p(t)$ on $[0, \infty)$ with $\mathcal{D}$ the subclass of $\mathfrak{b}$ for which $\int_{i}^{\infty} s p(s) d s=\infty$, and let $\mathcal{O}$ be the subclass of $b$ for which all solutions of (1.3) oscillate.

Then $\mathfrak{D}$ and $\mathcal{O}$ coincide if and only if there exist a monotone increasing, continuous function $f^{*}$ such that

$$
\left|f^{*}(y)\right| \leqslant|f(y)| \quad \text { for all } y
$$

and

$$
\int_{ \pm 1}^{ \pm \infty} \frac{d y}{f^{*}(y)}<\infty
$$

In particular, if $f$ is an unbounded monotone increasing function, with $\int_{-\infty}^{\infty} d y / f(y)=\infty$ (or $\int_{-1}^{-\infty} d y / f(y)=\infty$ ), then $\mathfrak{D} \neq \mathcal{O}$.

Proof. - That $\mathfrak{D}$ and $\mathcal{O}$ coincide if (3.1) is satisfied, is a consequence of the Theorem of Macki and Wong referred to in the introduction. To demonstrate that condition (3.1) is necessary, we consider first the case that $f$ is monotone increasing. Then the non-existence of a function $f^{*}$ with the required properties is (without loss of generality) equivalent to the statement $\int_{1}^{\infty} d y / f(y)=\infty$, and this case will therefore verify the final assertion of the Theorem.

Let $F(t)=\int_{0}^{t} f(u) d u, \Omega(t)=\int_{1}^{t} d u / f(u)$ (there is no loss of generality in assuming that $f(1)>0)$. We shall show that there exists

a positive, concave unbounded function $y(t)$ such that $y^{\prime \prime}=\frac{1}{2}[\Omega(y)]^{\prime \prime} f(y)$.

If we now define $p(t)$ to be $-\frac{1}{2}[\Omega(y)]^{\prime \prime}$, we have $\int_{i}^{T} p(s) d s=\frac{1}{2}[\Omega(y(t))]^{\prime}-\frac{1}{2}[\Omega(y(T))]^{\prime}$. Now $[\Omega(y(T))]^{\prime}=y^{\prime}(T) / f(y(T)) \rightarrow 0$ as $T \rightarrow \infty$ on account of $(3.2)$, and so $P(t)=$ $=\int_{i}^{\infty} p(s) d s=\frac{1}{2}[\Omega(y(t))]^{\prime}$. Integrating again we have

$$
\int_{i}^{t} P(s) d s=\frac{1}{2} \Omega(y(t))-\frac{1}{2} \Omega(y(1)) \rightarrow \infty \quad \text { as } t \rightarrow \infty \text {. }
$$


The non-negativity and integrability of $p(t)$ imply that $t P(t) \rightarrow 0$ as $t \rightarrow \infty$, and so we have

$$
\int_{i}^{\infty} s p(s) d s=t P(t)+\int_{i}^{\infty} P(s) d s=\infty,
$$

and the Theorem will be proved for monotone $f$. To obtain (3.2), we observe that the equation has a positive solution $y(t)$ if and only if $y$ solves the equation

$$
\frac{y^{\prime \prime}}{f(y)}+\frac{f^{\prime}(y) y^{\prime 2}}{f^{2}(y)}=0
$$

which, for positive $y$, is equivalent to

$$
\frac{d}{d t}\left(y^{\prime} f(y)\right)=0
$$

A suitable solution is found by solving

to obtain

$$
y^{\prime} f(y)=1, \quad y(0)=1
$$

$$
y(t)=F^{-1}(t+1)
$$

and this solution clearly satisfies (3.2).

In the general case, we need to consider two possibilities

$$
\text { (i) } \underline{\lim }_{y \rightarrow \infty} f(y)=\infty, \quad \text { (ii) } \underline{\underline{\lim }} f(y)<\infty
$$

If $(i)$ is the case, define $g(u)$ to be $\inf _{s \geqslant u} f(s)$.

Let $G(u)=\int_{0}^{u} g(s) d s, \quad \theta(u)=\int_{1}^{u} d s / g(s) \quad$ (we assume that $\left.g(1)>0\right)$. Then $g(u)$ is non-decreasing with $g(u) \leqslant f(u)$ and if (3.1) fails to hold, we may assume, without loss of generality, that

$$
\int_{1}^{\infty} \frac{d u}{g(u)}=\infty
$$

We note that $g(u) \rightarrow \infty$ as $u \rightarrow \infty$, and that $g$ is differentiable a.e.

Define $p(t)$ to be $-\frac{1}{2}[\theta(y(t))]^{\prime}$, where as before, $y$ will be a positive, unbounded, concave function satisfying

$$
\frac{y^{\prime \prime}}{f(y)}=\frac{1}{2}[\theta(y)]^{\prime \prime}, \quad \text { a.e. }
$$


(since $y$ will be concave, there will be no problems arising from the non-existence of $g^{\prime}$ on a null set). This equation may be written in the form

$$
\left\{\begin{aligned}
\frac{d}{d t}\left(y^{\prime} g(y)\right) & =0, & & g(y)=f(y) \\
y^{\prime \prime} & =0, & & g(y)<f(y)
\end{aligned}\right.
$$

and is solved (in the Carathéodory sense) by $y(t)=G^{-1}(t+1)$.

If (ii) is the case, the preceding construction fails, since it is no longer true that $[\Omega(y(T))]^{\prime}\left(\right.$ or $\left.[\theta(y(T))]^{\prime}\right)$ will tend to zero as $T \rightarrow \infty$. Instead, we define

$$
\tilde{f}(y)=\max (f(y), y), \quad y \geqslant 0 .
$$

Now let $\tilde{g}(y)$ be a continuous, monotone increasing function of $y$ with $0 \leqslant \tilde{g}(y) \leqslant \tilde{f}(y)$, $y \geqslant 0$. If $f(y) \leqslant y$ for all sufficiently large $g$, it is obvious that $\int_{i}^{\infty} d u / \tilde{g}(u)=\infty$; other-
wise let $u_{i} \uparrow \infty$ with $f\left(u_{i}\right)=u_{i}$.

Then for any natural numbers $m, n$ with $m<n$, we have

$$
\begin{aligned}
\int_{u_{m}}^{\infty} \frac{d u}{g(u)} & \geqslant \sum_{i=m}^{n} \int_{u_{i}}^{u_{i+1}} \frac{d u}{\tilde{g}(u)} \geqslant \sum_{i=m}^{n} \frac{u_{i+1}-u_{i}}{u_{i+1}} \\
& \geqslant \frac{1}{u_{n+1}}\left(u_{n+1}-u_{m}\right) \rightarrow 1 \quad \text { as } n \rightarrow \infty
\end{aligned}
$$

and it follows that $\int_{1}^{\infty} d u / \tilde{g}(u)=\infty$. Thus $\tilde{f}(y)$ falls under the category of case (i) previously considered. ( $\tilde{f}$ will be absolutely continuous, rather than $C^{1}$, but an examination of the proof yields that no essential change is required on this account) Hence there exists a function $p(t) \in \mathscr{D}$ such that

$$
y^{\prime \prime}+p(t) \tilde{f}(y)=0
$$

admits (in the Caratheodory sense) a non-trivial, non-oscillatory solution $y_{0}(t)$. Let $0<a<y_{0}(0)$, and consider the solutions of (1.3) with $y(0)=a, y^{\prime}(0)=b$, denoting them by $y(b ; t)$. (They are unique by the $\mathrm{C}^{1}$ hypothesis on $f$.) The solution curve $y(0 ; t)$ intersects the $t$-axis before it intersects the curve $y_{0}(t)$, and for $b$ sufficiently large, the solution curve $y(b ; t)$ intersects the curve $y_{0}(t)$ before it intersects the $t$-axis. Defining $b^{*}$ to be the supremum of those numbers $b$ for which the curve $y(b ; t)$ intersects the $t$-axis before it intersects the curve $y_{0}(t)$, it follows that $b^{*}$ is finite and nonnegative. Denote the solution $y\left(b^{*} ; t\right)$ by $y^{*}(t)$.

We shall show that $y^{*}$ is a nonoscillatory solution of $(1.3)$ on $(0, \infty)$. 
For suppose otherwise. Since $p(t) \geqslant 0$ for almost all $t$, solutions of (1.3) are concave in any interval in which they remain positive. Thus there exists $t_{1}>0$, such that $y^{*}\left(t_{1}\right)=0$. Using the definition of $b^{*}$, together with compactness and the sontinuous dependence of solutions of (1.3) on initial conditions, we may deduce the existence of a smallest positive value $t_{0}$ of $t$, for which $y^{*}\left(t_{0}\right)=y_{0}\left(t_{0}\right)=a_{0}$, say. Again, the continuity of solutions of (1.3) with respect to initial conditions impl es that $y^{*^{\prime}}\left(t_{0}\right)=y_{0}^{\prime}\left(t_{0}\right)$.

Now we define $F(t, u, v)$ to be $-p(t) f(u)$, and note that if $I=\left[0, t_{1}\right]$, $y_{0}(t)$ is positive on $I$ and $F$ is non-decreasing in $u$ and satisfies a uniform Lipschitz condition in $v$ everywhere on $I \times[0, \infty) \times(-\infty, \infty)$, with the exception of a threedimensional null set of planes parallel to the $(u, v)$ - plane.

Writing (1.3) in the form

$$
y^{\prime \prime}=F\left(t, y, y^{\prime}\right)
$$

we see that $y_{0}$ is an $A C^{(1)}$-upper solution of $\left(1.3^{\prime}\right)$ on $I$. Hence, by Corollary 3.6 of Theorem 2.5 of [5], to which we also refer for the appropriate definitions, it follows that $y_{0}$ is a superfunction with respect to solutions of $\left(1.3^{\prime}\right)$ on $I$, and so $y_{0} \leqslant y^{*}$ on $I$, which is a contradiction. Thus $y^{*}$ is nonoscillatory.

We should remark that in [5], it is assumed that $F(t, u, v)$ is continuous in all three variables, and is monotone in $u$ on $I \times R^{2}$. However, it requires no more than a routine modification of the proofs in that paper in order to apply the relevant theorems to $\left(1.3^{\prime}\right)$.

We have now dealt with case (ii), and so the proof of the Theorem is complete.

We note the following immediate

Corollary 3.1. - Let $\lim _{|y| \rightarrow \infty}|f(y)|<\infty$. Then $D \neq \mathcal{O}$. Theorem 3.1 may also be obtained under the restriction that the coefficients $p(t)$ are continuous (and that solutions of (1.3) are solutions for all $t$ ). The proof then requires modification via a number of technical lemmas, and so we merely state these and the restricted version of the Theorem.

LFima 3.1. - Let $f$ satisfy the hypotheses of Theorem 3.1, and let $g(u)=\inf _{s \geqslant u} f(s)$ Then $g^{\prime}$ may be redefined on a countable set to a function $\gamma$ whose discontinuities form a countable, nowhere dense set.

LEMMa 3.2. - Let $p(t) \in \mathcal{D}$ such that $p$ has only a countable, nowhere dense set of discontinuities. Then there exists $q(t) \in \mathfrak{D}^{\prime}$ (the restriction of $\mathfrak{D}$ to continuous functions) with $q(t) \leqslant p(t)$ for all $t \geqslant 0$.

Lemra 3.3. - Let $f$ satisfy the hypotheses of Theorem 3.1 such that $\frac{\lim _{|y| \rightarrow \infty}}{|y(y)|<\infty}$. Then there exists $\tilde{f}(y) \in C^{1}(-\infty, \infty)$ with $|f(y)| \leqslant|\tilde{f}(y)|$ for all $y$, and such that either $\int_{1}^{\infty} d u / \tilde{g}(u)$ or $\int_{-1}^{-\infty} d u / \tilde{g}(u)$ is infinite, for any monotone function $\tilde{g}(y)$ with $|\tilde{g}(y)| \leqslant|\tilde{f}(y)|$ for all $y$. 
Theorem 3.2. - Let $f$ satisfy the hypotheses of Theorem 3.1. Then the Theorem remains true if $\mathfrak{b}, \mathfrak{D}, \mathcal{O}$, are replaced by their restrictions $\mathfrak{b}^{\prime}, \mathfrak{D}^{\prime}, \mathcal{O}^{\prime}$, respectively, to continuous functions.

We briefly turn our attention to the condition that $f^{\prime}(y)$ should be bounded away from zero on closed sets that exclude $y=0$. It is easy to demonstrate that $f$ cannot be allowed to be constant on any interval; for, without loss of generality, suppose that $f \equiv 1$ on $[a, b]$, where $0<a<b<\infty$. Let $p(t)$ be any locally integrable function for which $\int_{t}^{\infty} p(s) d s=P(t)$ exists for all $t$, and such that $\left|\int_{t}^{\infty} P(s) d s\right|<\infty$ and $\int_{i}^{\infty} \int_{s}^{\infty} P^{2}(\sigma) d \sigma d s \stackrel{i}{=} \infty$

$$
\begin{aligned}
& \text { Ohoose } t_{0} \text { so that }\left|\int_{i}^{\infty} P(s) d s\right|<(b-a) / 2 \text { for all } t \geqslant t_{0}, \text { and then define } y(t) \text { for } t \geqslant t_{0} \text {, by } \\
& \qquad y(t)=\frac{a+b}{2}-\int_{t}^{\infty} P(s) d s .
\end{aligned}
$$

We have $y^{\prime \prime}(t) \geqslant-p(t) \geqslant-f(y(t)) p(t)$, and so $y(t)$ is a non-oscillatory solution of (1.3). The precise status of the condition on $f^{\prime}(y)$ seems unclear.

\section{4. - Some examples.}

We illustrate our theorems with some examples which do not seem to be covered by results of earlier authors. The relevant conditions on $p(t)$ are easily verified

1) Let

$$
p(t)=\frac{\mu \cos v t}{t}+\frac{(1+\sin v t)}{t^{2}} \quad(\mu, v \text { non-zero constants }) .
$$

Suppose that $|\mu| \nu \mid>1$ Then $P(t)$ alternates in sign, but $\int_{i}^{\infty} P(s) d s=\infty$ and so all
extendable solutions of $(1.3)$ oscillate (Theorem 2.1 ).

2) Let

$$
p(t)=\frac{\mu \cos v t}{t(\log t)^{\frac{1}{2}+\delta}}
$$

$\left(\mu, v\right.$ non-zero and $\left.\varepsilon \geqslant-\frac{1}{2}\right)$.
$P(t)$ alternates in sign and $\left|\int_{i}^{\infty} P(s) d s\right|<\infty$. If $\varepsilon \leqslant 0$, we have $\int_{t}^{\infty} \int_{s}^{\infty} P_{+}^{2}(\sigma) d \sigma d s=\infty$ and so all extendable solutions of (1.3) oscillate (Theorem 2.1); if $\varepsilon>0$, both $\int_{t}^{\infty} \int_{s}^{\infty} P_{+}^{2}(\sigma)$. $\cdot d \sigma d s$ and $\int_{t}^{\infty} \int_{s}^{\infty} P_{-}^{2}(\sigma) d \sigma d s$ are convergent integrals, and so (1.3) has a non-oscillatory solution (Theorem 2.2). 
3) In this example, it will be convenient to define a piecewise continuous function $P(t)$ which tends to zero as $t$ approaches infinity. It is not difficult, although tedious, to construct a smooth function $P(t)$ with the same integral properties and then define $p(t)$ to be $-P^{\prime}(t)$.

Let $\alpha, \beta>0$ and, for sufficiently large $t$, define

$$
P(t)= \begin{cases}n^{-\alpha}, & n \leqslant t \leqslant n+n^{-\beta} \\ 0, & \text { otherwise }\end{cases}
$$

Then if $\alpha+\beta>1$, we have $\int_{i}^{\infty} P(s) d s<\infty$. If, in addition, we have $2 \alpha+\beta \leqslant 2$, then $\int_{i}^{\infty} \int_{s}^{\infty} P_{+}^{2}(\sigma) d \sigma d s=\infty$ and so extendable solutions of (1.3) oscillate; if the inequality is reversed then the iterated integral converges and there is a non-oscillatory solution.

4) Let

$$
\begin{aligned}
& a_{n}=n+\frac{1}{n^{2}}, \quad b_{n}=n+1-\frac{1}{(n+1)^{2}}, \\
& c_{n}=\frac{1}{2}\left(a_{n}+b_{n}\right), \quad a_{n}=\left(b_{n}-a_{n}\right), \quad n=1,2, \ldots .
\end{aligned}
$$

Define $p$ by

$$
p(t)= \begin{cases}\frac{1}{2}\left(b_{n}-a_{n}\right)-\left|t-c_{n}\right|, & a_{n} \leqslant t \leqslant b_{n} \\ -\frac{1}{2} d_{n-1} n^{2}+d_{n-1} n^{4}|t-n|, & b_{n-1}<t<a_{n}\end{cases}
$$

Then $\int_{t}^{\infty} p(s) d s$ does not exist. However conditions $\left(\pi_{3}\right),\left(\pi_{4}\right)$ and $\left(\pi_{5}\right)$ of Theorem 2.4 are satisfied and $\int_{i}^{\infty} \hat{P}(s) d s=\infty$. Hence all solutions of (1.3) oscillate.

As a final remark, it would be of some interest to have conditions on the coefficient $p(t)$ which would guarantee the existence of non-trivial extendable solutions to (1.3). Burton and Grimmer [2] have shown that if $p(t)$ is continuous and takes negative values, then there are non-extendable solutions. In the other direction, KIGURADZE [6] has a partial and somewhat complicated condition that implies the existence of an extendable solution.

\section{REFERENCES}

[1] F. V. Atkrnson, On second order nonlinear oscillation, Pacific J. Math., 5 (1955), pp. 643-647.

[2] T. Burton - R. GRTMmer, On continuability of solutions of second order differential equations, Proc. Amer. Math. Soc., 29 (1971), pp. 277-283. 
[3] W. J. CoLes, Oscillation criteria for nonlinear second order equations, Ann. di Mat. Pura ed Appl., 82 (4) (1969), pp. 123-134.

[4] L. H. ERBE, Oscillation theorems for second order nonlinear differential equations, Proc. Amer. Math. Soc., 24 (4) (1970), pp. 811-814.

[5] L. K. JACKSON, Subfunctions and second-order ordinary differential inequalities, Advances in Math., 2 (1968), pp. 307-363.

[6] I. T. KIGURAdZE, A note on the oscillation of $u^{n}+a(t)|u|^{n}$ sgn $u=0$, Casopis Pest. Math., 92 (1967), pp. 343-350 (Russian).

[7] J. W. MACKI, An example in the theory of nonlinear oscillations, SIAM J. Appl. Math., 17 (3) (1969), pp. 517-519.

[8] J. W. MACKI - J. S. W. WONG, Oscillation of solutions to second-order nonlinear differential equations, Pacific J. Math., 24 (1) (1968), pp. 111-117.

[9] R. A. Moore - Z. NEHARI, Non-oscillation theorems for a class of nonlinear differential equations, Trans. Amer. Math. Soc., 93 (1959), pp. 30-52.

[10] Z. NEHARI, On a class of nonlinear sewond order differential equations, Trans. Amer. Math. Soc., 95 (1960), pp. 101-123.

[11] W. R. UTz, Properties of solutions of $u^{\prime \prime}+g(t) u^{2 n-1}=0$, Monatsh. Math., 66 (1962), pp. 55-60.

[12] W. R. UTz, Properties of solutions of $u^{\prime \prime}+g(t) u^{2 n-1}=0$ (II), Monatsh. Math., 69 (1965), pp. $353-361$.

[13] P. Waltman, Some properties of $u^{\prime \prime}+a(t) f(u)=0$, Monatsh. Math., 67 (1963), pp. 50-54.

[14] P. Waltman, An oscillation criterion for a nonlinear second order equation, J. Math. Anal. Appl., 10 (1965), pp. 439-441.

[15] D. WILLETT, On the oscillatory behaviour of the solutions of second order linear differential equations, Ann. Polon. Math., 21 (1969), pp. 175-194.

[16] J. S. W. WONG, A note on second order nonlinear oscillation, SIAM Review, 10 (1968), pp. 88-9l.

[17] J. S. W. Won, On second order nonlinear oscillation, Funke. Ekv,, 11 (1968), pp. 207-234. 